\title{
Effect of Thromboxane Synthetase Inhibition on Canine Autogenous Vein Grafts
}

\author{
Eric D. Endean, M.D., JefFrey M. Boorstein, B.S., Paul L. Hees, B.S., \\ AND JACK L. CRONENWETT, M.D. ${ }^{1}$ \\ Department of Surgery, University of Michigan Medical Center, Ann Arbor, Michigan 48109, \\ and Dartmouth-Hitchcock Medical Center, Hanover, New Hampshire 03756
}

Presented at the Annual Meeting of the Association for Academic Surgery, Cincinnati, Ohio, November 10-13, 1985

\begin{abstract}
This study examined the effect of an orally active thromboxane (TXA $)$ synthetase inhibitor (TSI) on the patency, $\mathrm{TXA}_{2}$ production, and platelet accumulation of reversed autogenous vein grafts. Ten dogs received TSI (U-63557A) $10 \mathrm{mg} / \mathrm{kg}$ po $\mathrm{q} 8 \mathrm{hr}$ for 6 weeks, beginning $24 \mathrm{hr}$ prior to surgery, while 15 control dogs were untreated. One jugular vein was harvested and stored in $37^{\circ} \mathrm{C}$ saline for $1 \mathrm{hr}$ to induce mild endothelial injury (stored). Normal and stored jugular vein grafts $(8 \mathrm{~cm})$ were then implanted in opposite femoral arteries while $3-\mathrm{cm}$ segments of the same veins were implanted in the carotid arteries. Femoral graft flow was restricted with a $5 \mathrm{Fr}$ distal arterial stenosis and patency determined by arteriography at $1,2,4$, and 6 weeks. Vein graft endothelial surface $\mathrm{TXB}_{2}$ production was measured by RIA at graft implantation and in carotid grafts harvested at 1 week. ${ }^{111}$ In-labeled platelets were given iv 24 hr prior to carotid graft harvest to determine graft-platelet deposition. TSI treatment improved early (1 week) femoral vein graft patency from 63 to $89 \%(P<0.05)$, a trend that persisted for 6 weeks. Warm saline storage reduced 1-week graft patency from 83 to $63 \%(P<0.05)$, a difference that decreased with time. TSI treatment resulted in a marked decrease in $\mathrm{TXB}_{2}$ production, but was not associated with decreased "In-labeled platelet deposition in carotid vein grafts. Warm saline storage increased graft-platelet deposition which was predominant at the arterial anastomoses. TSI treatment may improve early vein graft patency during the transient period of endothelial injury. (c) 1986 Academic Press, Inc.
\end{abstract}

\section{INTRODUCTION}

Aspirin is frequently used as an antiplatelet agent in an attempt to improve the patency rate of small diameter vascular grafts. Such cyclooxygenase inhibitors, however, have the theoretical disadvantage of inhibiting prostacyclin $\left(\mathrm{PGI}_{2}\right)$ production in addition to thromboxane $\mathrm{A}_{2}\left(\mathrm{TXA}_{2}\right)$ synthesis. This criticism has stimulated the development of selective thromboxane synthetase inhibitors (TSIs) which may increase $\mathrm{PGI}_{2}$ production by redirecting endoperoxide precursors that accumulate during $\mathrm{TXA}_{2}$ inhibition [11]. Since autogenous vein grafts retain the ability to synthesize $\mathrm{PGI}_{2}[6]$, we hypothesized that TSI treatment would be more beneficial for these grafts than for prosthetic grafts. This

\footnotetext{
I To whom reprint requests should be addressed: Department of Surgery, Dartmouth College, Hanover, New Hampshire 03756.
}

study was designed to test the ability of a new TSI, U-63557A [24], to enhance the patency rate of autogenous vein grafts in the canine femoral artery circulation. The in vivo production of $\mathrm{TXB}_{2}$ (the stable metabolite of $\mathrm{TXA}_{2}$ ) and platelet accumulation on vein graft surfaces were measured to correlate with the effect of U-63557A on graft patency.

\section{METHODS}

Graft patency determination. Twenty-five adult mongrel dogs of both sexes $(18-30 \mathrm{~kg}$ ) were divided into control $(n=15)$ and treatment $(n=10)$ groups. Treatment dogs received U-63557A [sodium 5-(3'-pyridinylmethyl)benzofuran-2-carboxylate], an orally active TSI, $10 \mathrm{mg} / \mathrm{kg}$ po q8 hr beginning 24 hr prior to surgery and continuing for the 6week duration of study. Control dogs received no treatment. After iv thiamylal induction, 
dogs were anesthetized with halothane, nitrous oxide, and oxygen inhalation for graft implantation. Using sterile technique, the femoral and carotid arteries and external jugular veins were exposed bilaterally. Both jugular veins were harvested to provide an 8-cm segment for superficial femoral artery (SFA) implantation in all dogs; a 3-cm segment for common carotid artery implantation in six treated and eight control dogs; and a $2-\mathrm{cm}$ segment for baseline $\mathrm{TXB}_{2}$ assay in all dogs. One of the jugular veins was removed for immediate implantation without storage, distention, or irrigation, while the contralateral jugular vein was placed in $37^{\circ} \mathrm{C}$ normal saline storage for $1 \mathrm{hr}$ in an attempt to induce endothelial damage. Vein grafts placed in the femoral location were reversed and anastomosed in an end-to-side fashion using continuous 6-O polypropylene suture with spatulation of the anastomoses, from the proximal SFA, immediately distal to the profunda branch, to a point in the SFA $8 \mathrm{~cm}$ distally. A 5 Fr ligature was placed around the distal SFA in order to reproducibly restrict graft outflow. The mid portion of the SFA was ligated to completely divert blood flow through the vein graft. Vein grafts placed in the common carotid arteries were reversed and anastomosed in an end-to-end fashion using continuous 6-O polypropylene suture. Femoral and carotid vein grafts were simultaneously implanted by two operating teams to ensure equivalent graft treatment and storage time. The jugular vein removed for immediate use (normal vein) was implanted on one side in both the carotid and femoral locations, whilc the jugular vein selected for 1 -hr warm saline storage (stored vein) was implanted into the contralateral carotid and femoral artery locations.

A midline, lower abdominal incision was used to expose the middle sacral artery. An INFUSAID injection port was implanted in a subcutaneous pocket in the lower abdomen and its Silastic catheter placed just inside the infrarenal aorta via the middle sacral artery for subsequent arteriography. The surgical incisions were closed and the dogs recovered from anesthesia. Normal saline (1-1.5 liv) was administered during the 3 -hr operative procedure, and penicillin-G plus streptomycin were administered im preoperatively and on Postoperative Days 1 and 2.

Arteriograms were obtained under brief thiamylal sodium anesthesia at $24 \mathrm{hr}$ and at $1,2,4$, and 6 weeks postoperatively. Renografin-76 $(20 \mathrm{ml})$ was injected into the infrarenal aorta via the subcutaneous injection port and a single timed X-ray exposure was made to visualize the femoral artery grafts.

Graft platelet deposition. The subset of dogs that received carotid vein grafts underwent indium-111 oxinc platclet labcling on Postoperative Day 6. Platelet labeling was performed using a modification of the technique of Thakur et al. [23]. Venous blood was drawn through a large bore needle into plastic syringes containing two different anticoagulants ( $36 \mathrm{ml}$ blood: $4 \mathrm{ml}$ acid citrate dextrose (ACD); $18 \mathrm{ml}$ blood: $2 \mathrm{ml} 3.8 \%$ sodium citrate). After centrifugation of these separate samples at $200 \mathrm{~g}$ for $10 \mathrm{~min}$, platelet-rich plasma (PRP) and platelet-poor plasma (PPP) were isolated. Both ACD and sodium citrate-prepared PRP were then centrifuged at $100 \mathrm{~g}$ for $10 \mathrm{~min}$. Traces of ACD plasma in the platelet button were eliminated by twice layering and removing $2 \mathrm{ml}$ of modified Tyrodes solution (MTS) [23] at $37^{\circ} \mathrm{C}$. Platelet pellets were then suspended in $4 \mathrm{ml} \mathrm{MTS}$ at $37^{\circ} \mathrm{C}$ and $500 \mathrm{mCi}$ $(0.5 \mathrm{ml})$ of ${ }^{111} \mathrm{In}$ oxine (Amersham, saline solution) was added dropwise to the platelet suspension. Platelets were then incubated at $37^{\circ} \mathrm{C}$ for $10 \mathrm{~min}$, and $4 \mathrm{ml}$ of ACD-prepared PPP was added prior to 7 -min centrifugation at $1000 \mathrm{~g}$ to remove any unbound oxine. The labeled platelets were finally resuspended in 4 $\mathrm{ml}$ of sodium citrate-prepared PPP for iv reinjection into the animal.

One day later, the dogs were reanesthetized (thiamylal sodium), the neck incisions reexplored, and the carotid vein grafts harvested to include a $1-\mathrm{cm}$ segment of proximal and distal native carotid artery. After removal, the graft segments and adjacent carotid artery were gently flushed with normal saline to remove any contained blood. The grafts were then di- 
vided into five segments, $1 \mathrm{~cm}$ in length, consisting of proximal and distal native carotid artery, proximal and distal anastomoses, and central vein graft. Each sample was weighed and subsequently counted for ${ }^{111}$ In activity in a gamma well counter. A sample of blood was removed at the time of carotid graft harvest and also counted to determine the ${ }^{111}$ In activity of circulating platelets. Since graft and artery diameters were uniform in these dogs, small variations in the length of the counted graft and artery segments were normalized by dividing the counts per segment by the segment weight. Platelet deposition was expressed as the ratio of the ${ }^{11}$ In counts per gram of graft (artery) segment divided by the counts per $100 \mu \mathrm{l}$ of circulating blood.

Thromboxane production. Luminal vein graft $\mathrm{TXB}_{2}$ production was measured using a technique suggested by Eldor $e$ al. [12]. The segment of vein to be analyzed was incised longitudinally to expose the endothelial surface, which was gently but thoroughly irrigated with Tris buffer ( $\mathrm{pH}$ 7.4). The vein segment was then clamped in a plastic template which exposed a $0.79-\mathrm{cm}^{2}$ endothelial surface area to a 2-cm-deep circular well which was filled with $1 \mathrm{ml}$ Tris buffer at $37^{\circ} \mathrm{C}$. The vein graft was incubated in this template for $30 \mathrm{~min}$ at $37^{\circ} \mathrm{C}$, after which the buffer was removed and frozen for subsequent $\mathrm{TXB}_{2}$ radioimmunoassay [13]. Vein graft $\mathrm{TXB}_{2}$ production was measured at the time of graft implantation from both immediately implanted and warm saline-stored graft segments, as well as from vein grafts harvested after 1 week of carotid implantation. The central portion of the carotid vein graft was used for this determination prior to the determination of its ${ }^{11}$ In activity.

Statistical analysis. Vein graft patency was analyzed by product moment actuarial technique with significant differences determined by the Wilcoxon test. Patency at individual time points for TSI-treated and control dogs was tested by the Fisher exact test for independent samples, while differences between immediately implanted and warm salinestored grafts were tested by the McNemar test for paired data [9]. $\mathrm{TXB}_{2}$ production and ${ }^{1 " I} \mathrm{In}$ platelet deposition were compared between treatment groups by the Mann-Whitney test and within treatment groups by the Wilcoxon sign ranks test (all two-tail analyses) [9]. Correlation between graft patency, $\mathrm{TXB}_{2}$ production, and platelet deposition was measured by the Spearman rank correlation coefficient.

\section{RESULTS}

In this canine model, autogenous jugular vein grafts placed in the femoral artery location demonstrated 6-week cumulative patency rates of 53 to $70 \%$. Most graft occlusions occurred during the first 2 weeks of implantation, with only 2 of 50 grafts occluding between 2 and 6 weeks (Fig. 1). TSI treatment appeared to improve the patency of both normal and warm saline stored vein grafts when compared with untreated control dogs, although actuarial analysis of these survival curves did not demonstrate statistical significance (Wilcoxon test). The effect of TSI was most apparent 1 week after graft implantation when normal vein grafts in control dogs demonstrated a $27 \%$ lower patency than in TSI-treated dogs $(P$ $=0.13$, Fisher exact test, Table 1). This nearly significant difference also existed for saline stored grafts, which had a $27 \%$ lower 1-week patency in control dogs compared with TSItreated dogs $(P=0.20$, Fisher exact test, Table 1). In addition to the effect of drug treatment

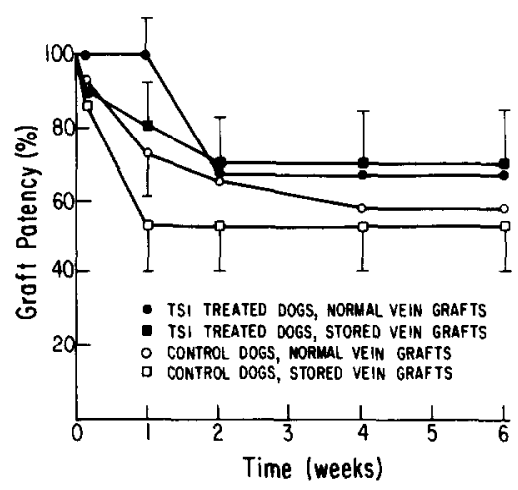

FIG. 1. Femoral artery autogenous vein graft patency in 15 control and 10 thromboxane synthetase inhibitor (TSI)-treated dogs (means \pm SEM). Normal and warm saline-stored vein grafts were placed in contralateral sides in each animal. 
TABLE 1

Femoral Artery autogenous Ven Graft Patency (\%)

\begin{tabular}{|c|c|c|c|c|}
\hline & \multicolumn{2}{|c|}{1 week } & \multicolumn{2}{|c|}{6 weeks } \\
\hline & Normal vein & Stored vein & Normal vein & Stored vein \\
\hline \multirow[t]{2}{*}{ Control } & \multicolumn{2}{|c|}{$P=0.20^{* *}$} & & \\
\hline & $P=0.12 *$ & $P=\stackrel{1}{1}_{0.19 *}^{53 \%}$ & $\prod_{n}^{58 \%}$ & $\int_{n *}^{53 \%}$ \\
\hline TSI treated & $100 \%$ & $80 \%$ & $70 \%$ & $67 \%$ \\
\hline & $L$ & & & \\
\hline
\end{tabular}

* Fisher exact test for independent samples (two tailed).

** McNemar test for paired data (two tailed).

on graft patency, warm saline storage reduced 1-week graft patency by $20 \%$ in both TSItreated and control dogs. These differences, which approached statistical significance at 1 week, were nearly eliminated by 6 weeks, when normal and saline-stored veins had comparable patency rates (Table 1).

Graft and treatment subgroups were combined to evaluate the effect of TSI treatment on both normal and saline-stored vein grafts ( $n=30$ control, $n=20$ treatment), as well as the effect of warm saline storage on both control and TSI treated dogs $(n=25$ normal, $n$ $=25$ stored vein grafts). TSI treatment improved early ( 1 week) vein graft patency from 63 to $89 \%$ (Fig. 2, $P<0.05$, Fisher exact test). Although this effect persisted for 6 weeks, the difference in overall survival curves only approached statistical significance $(P=0.21$, Wilcoxon test). Warm saline storage reduced 1-week graft patency from 83 to $63 \%$ (Fig. 3, $P<0.05$, McNemar test). This difference subsequently decreased with time, however, so that no difference existed between normal and saline-stored grafts at 6 weeks $(P=0.31$, Wilcoxon test).

Vein graft $\mathrm{TXB}_{2}$ production was three to seven times lower in TSI-treated dogs than in control dogs when assayed at implantation and at 1 week postoperatively (Table 2). These reductions were highly significant for both normal and warm saline-stored grafts. $\mathrm{TXB}_{2}$ pro- duction increased three- to sevenfold in all vein grafts removed at 1 week compared with the same grafts at implantation. There was no significant difference in $\mathrm{TXB}_{2}$ production, however, between normal and saline-stored vein grafts at implantation or at 1 week postoperatively (Table 2 ).

${ }^{111}$ In-labeled platelet deposition occurred predominantly at the proximal and distal carotid vein graft anastomoses. There was no significant difference in platelet deposition between the proximal and distal carotid artery segments and the midportion of normal jugular vein grafts in control dogs (Table 3). Significantly increased platelet deposition did oc-

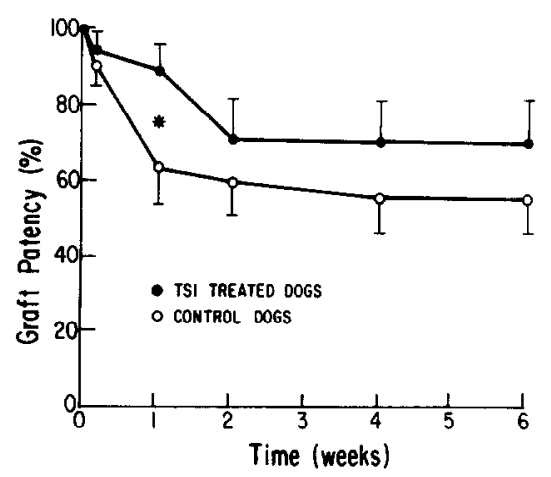

FIG. 2. Patency of all femoral vein grafts (both normal and saline stored) in control versus thromboxane synthetase inhibitor (TSI)-treated dogs (means \pm SEM). At 1 week, ${ }^{*} P<0.05$, Fisher exact test. 


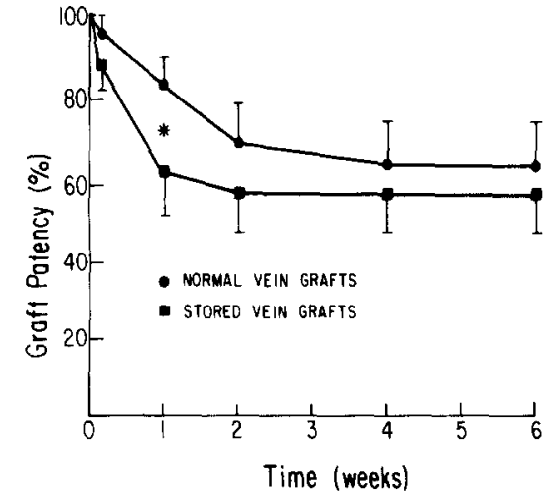

FIG. 3. Patency of normal versus warm saline-stored autogenous vein grafts in the femoral artery position of both control and TSI-treated dogs (means \pm SEM). At 1 week, ${ }^{*} P<0.05$, McNemar test.

cur at both anastomoses. Platelet deposition was two to four times greater in saline-stored vein grafts in the same control dogs, at both the anastomoses and the vein graft itself (Table 3 ). Dogs treated with TSI also demonstrated increased platelet deposition at the anastomoses, but also in the vein graft itself, when compared with proximal or distal artery segments. This enhanced platelet accumulation in normal vein grafts of TSI-treated dogs was significantly greater than the mimimal deposition seen in control dogs $(P<0.02$, MannWhitney test). No other significant differences in platelet deposition existed between control and TSI treated animals. As with control dogs, saline-stored vein grafts demonstrated in- creased platelet deposition in both the graft and the anastomoses when compared with proximal or distal native artery (Table 3 ).

There was no correlation $\left(r_{\mathrm{s}}<0.2\right)$ between platelet deposition (vein graft or anastomoses) and $\mathrm{TXB}_{2}$ production at the time of carotid vein graft harvest. Furthermore, there was no correlation between vein graft $\mathrm{TXB}_{2}$ production or platelet deposition and 1- or 6-week patency of comparably treated femoral artery grafts in the same animals.

\section{DISCUSSION}

In contrast to aspirin and other cyclooxygenase inhibitors, selective inhibition of $\mathrm{TXA}_{2}$ synthetase has the theoretical advantage of preserving or increasing $\mathrm{PGI}_{2}$ production. Accumulated endoperoxides resulting from $\mathrm{TXA}_{2}$ inhibition appear to be redirected to enhance $\mathrm{PGI}_{2}$ synthesis when $\mathrm{PGI}_{2}$ synthetase is available $[8,10,11]$. Despite this potential advantage, TSIs do not inhibit in vitro platelet aggregation as consistently as aspirin [8]. This may be due to the proaggregatory activity of certain endoperoxide intermediates that do not accumulate after cyclooxygenase inhibition [10]. These considerations suggest that selective $\mathrm{TXA}_{2}$ inhibition would have maximum advantage over cyclooxygenase inhibition in conjunction with autogenous tissue that is capable of $\mathrm{PGI}_{2}$ generation.

Despite these theoretical objections, aspirin

TABLE 2

ThromboXane $B_{2}$ Production (ng/0.79 $\mathrm{cm}^{2}$ Vein Surface $/ 30 \mathrm{~min}$ )

\begin{tabular}{|c|c|c|c|c|c|c|}
\hline \multirow[b]{2}{*}{ Time } & \multicolumn{3}{|c|}{ Normal vein grafts ${ }^{a}$} & \multicolumn{3}{|c|}{ Stored vein grafts ${ }^{a}$} \\
\hline & Control & TSI treated & $P$ value ${ }^{b}$ & Control & TSI treated & $P$ value $^{b}$ \\
\hline Implantation & $\prod_{l}^{431 \pm 58}$ & $\stackrel{129 \pm 57}{!}$ & $<0.002$ & $308 \pm 58$ & $\prod_{*}^{112 \pm 21}$ & $<0.002$ \\
\hline 1 week postoperative & $2727 \pm 1065$ & $378 \pm 86$ & $<0.003$ & $2266 \pm 545$ & $555 \pm 141$ & $<0.01$ \\
\hline
\end{tabular}

Note. Values are means $\pm \mathrm{SEM}$.

* $P<0.03$, Implantation vs 1 week, Wilcoxon sign ranks test.

a Normal vs stored vein grafts, no significant differences, Wilcoxon sign ranks test.

${ }^{b}$ Control vs TSI-treated dogs, Mann-Whitney test. 
TABLE 3

PlateleT-GRAFt DePOSITION (COUNTS ${ }^{11}$ In/g GRAFT $\div$ COUNTS ${ }^{11}$ In/ $100 \mu 1$ BLOOD)

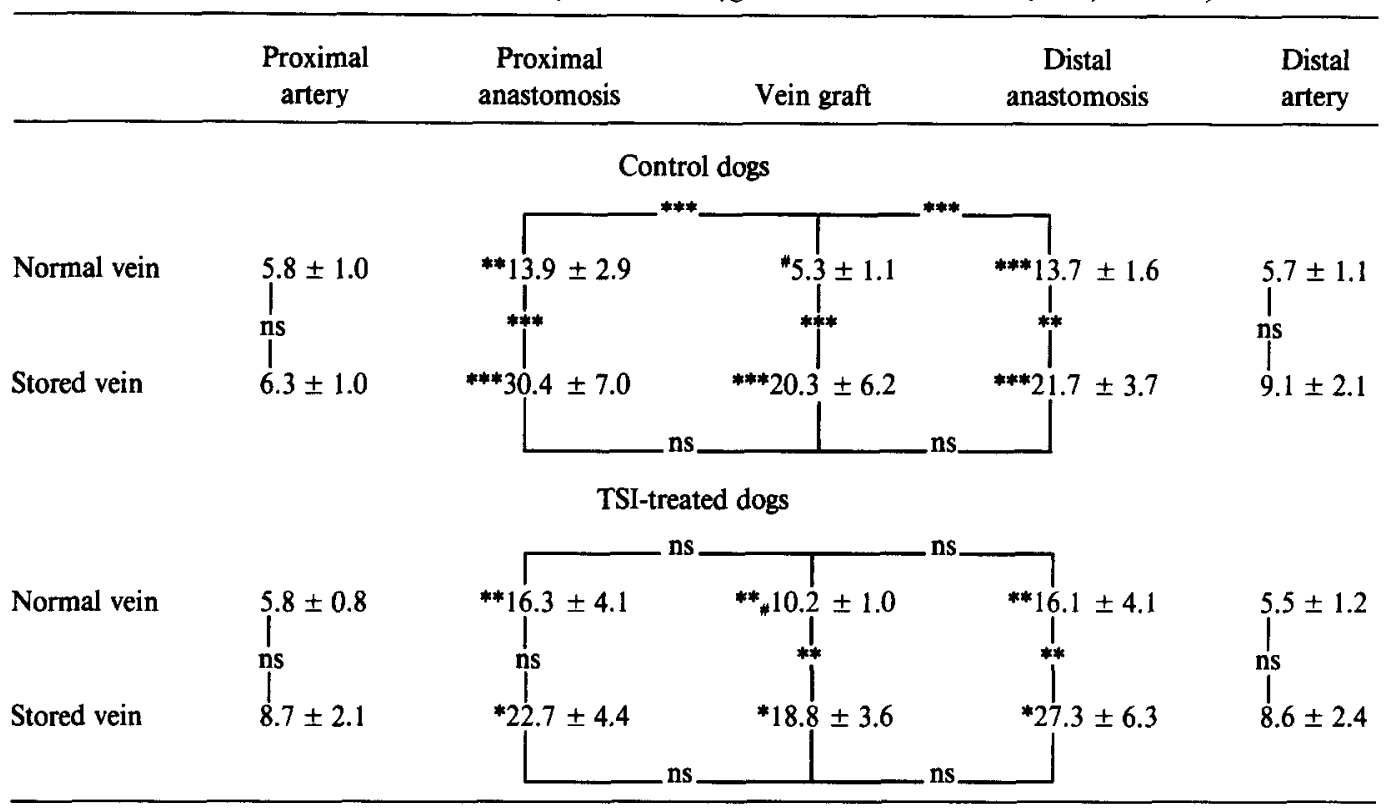

${ }^{*} P<0.10,{ }^{* *} P<0.05,{ }^{* * *} P<0.025$, Wilcoxon sign-ranks test (two tail). $\# P<0.02$, control vs TSI-treated dogs for normal vein grafts, Mann-Whitney test. Other control vs treated dog comparisons all nonsignificant $(P>0.10)$.

(often combined with dipyridamole) has improved aortocoronary vein graft patency in some clinical trials and has reduced vein graft anastomotic intimal fibroplasia in experimental models $[6,20]$. In contrast, aspirin treatment in rabbit endothelial injury models inhibited endothelial healing, enhanced platelet accumulation, and reduced $\mathrm{PGI}_{2}$ production $[3,4]$. Treatment of injured rabbit endothelium with TSI, however, reduced platelet deposition and enhanced $\mathrm{PGI}_{2}$ synthesis $[10,16$, 21]. Furthermore, aspirin-dipyridamole did not increase the patency rate of lower extremity autogenous vein grafts in humans [19]. Since vein graft occlusion remains a significant clinical problem, the above considerations suggested our study of the effect of selective $\mathrm{TXA}_{2}$ inhibition on vein graft patency.

Although TSI caused a marked reduction of $\mathrm{TXB}_{2}$ production in our study, its ability to improve vein graft patency was only modest. Furthermore, even though improved early graft patency was seen in TSI-treated dogs, no corresponding reduction in graft-platelet deposition was demonstrated. Since vein grafts do not accumulate sufficient ${ }^{111}$ In-labeled platelets for external gamma camera imaging, it was necessary to remove vein grafts to measure platelet deposition in a well counter. This prevented the measurement of graft patency and platelet deposition in the same graft and led to the use of carotid and femoral artery sites in our model. In retrospect, differences in anastomotic technique, graft length, and blood flow rate might have caused predictable differences in platelet deposition between femoral and carotid artery grafts. Although we did not measure graft flow rates, femoral outflow stenosis almost certainly caused signifcantly lower graft blood flow in the femoral than in the carotid location. Since lower flow rates increase platelet graft deposition [7], measurement of platelet deposition in the femoral grafts themselves might have correlated better with early improved femoral graft patency in TSI-treated dogs. These results, 
therefore, should not be interpreted as disproving a relationship between TSI effects on graft patency and a potential anti-platelet mechanism.

Our finding of improved early vein graft patency after TSI treatment is supported by a recent report of Bergquist $e t$ al. [2]. These investigators demonstrated that dazoxiben, an imidozole-derived TSI, improved the patency of human umbilical vein grafts after $4 \mathrm{hr}$ of ex vivo perfusion in sheep. Decreased platelet deposition and thrombus weight were associated with decreased $\mathrm{TXB}_{2}$ production. Dazoxiben and other TSIs have also been shown to reduce platelet adhesion on endothelium damaged rabbit arteries and to reduce $\mathrm{TXB}_{2}$ but increase $\mathrm{PGI}_{2}$ production [10, 21]. A significant effect of TSI treatment was not seen with Dacron grafts, however, where dazoxiben failed to reduce platelet deposition in an $e x$ vivo perfusion circuit or in baboon $\mathrm{A}-\mathrm{V}$ shunts, despite reduced $\mathrm{TXB}_{2}$ production $[14,17]$. These observations suggest that endogenous $\mathrm{PGI}_{2}$ production, not present in prosthetic grafts, may be essential for TSI efficacy. This has been demonstrated in a canine coronary artery stenosis model in which effective TSI prevention of platelet aggregation was blocked by cyclooxygenase inhibition that prevented $\mathrm{PGI}_{2}$ production $[1,15]$. U-63557A, the TSI used in this study, has been shown to signifcantly reduce $\mathrm{TXB}_{2}$ production in dogs, monkeys, and humans without tachyphylaxis after 14 days of oral administration [15]. A dose of $10 \mathrm{mg} / \mathrm{kg}$ markedly reduced $\mathrm{TXB}_{2}$ production in dogs for 4 to $6 \mathrm{hr}$ [24]. Recent experience in our laboratory suggests that a larger dose of U-63557 A might have had more beneficial effects in this study.

Vein graft storage in $37^{\circ} \mathrm{C}$ saline was used in our study to produce a mild endothelial injury analogous to some clinical settings. LoGerfo et al. reported that such storage resulted in early endothelial disruption and platelet-leukocyte adhesion, with subsequent medial thickening [18]. Although we did not examine these morphologic changes, warm saline storage was associated with reduced vein graft patency and increased platelet accumulation 1 week after implantation, during the time when endothelial injury exists, according to LoGerfo et al. Elimination of the saline storage effect on 4- to 6-week graft patency corresponds to the time course of endothelial healing after this degree of vein injury [18]. The basis for increased $\mathrm{TXB}_{2}$ production after 1 week of vein graft implantation was not specifically investigated in this study. We speculate that this increased production may have resulted from increased luminal accumulation of platelets, monocytes, and leukocytes during the first postoperative week that were not present when vein grafts were implanted. Although arteries and veins have been shown to synthesize $\mathrm{TXA}_{2}$ in addition to their more predominant $\mathrm{PGI}_{2}$ production [22], electron microscopy of the vein graft luminal surface would be necessary to exclude a major $\mathrm{TXA}_{2}$ contribution from adherent blood elements.

The use of femoral artery outflow stenosis to reduce autogenous vein graft patency resulted in a 6-week graft patency rate of 53 to $58 \%$ in control dogs. Since no grafts occluded between 4 and 6 weeks, this appears to be a practical model to investigate vein graft patency in a 4-week study in dogs. Such a technique is necessary since unrestricted flow through canine autogenous vein grafts nearly always results in $100 \%$ long-term patency, making such an animal model impractical for a study with graft occlusion as the endpoint.

Although it is generally accepted that platelet-graft interaction is an important contributor to arterial graft failure, it is equally clear that many factors influence early and late graft patency. Since we do not yet have data concerning the ability of aspirin or other cyclooxygenase inhibitors to improve the patency of vein grafts in this particular animal model, it is difficult to judge the significance of the improvement seen with TSI in this report. The ability of TSI to improve early vein graft patency, during the period of maximal, but transient endothelial injury and decreased $\mathbf{P G I}_{2}$ production, however, might be important. These results should stimulate a comparison 
of the chronic effects of both TSI and aspirin on graft patency and platelet deposition in an appropriate animal model.

\section{ACKNOWLEDGMENTS}

This study was supported in part by NIH grant HL35102. U-63557A was provided by the Upjohn Company.

\section{REFERENCES}

1. Aiken, J. W., Shebuski, R. J., Miller, O. V., and Gorman, R. R. Endogenous prostacyclin contributes to the efficacy of a thromboxane synthetase inhibitor for preventing coronary artery thrombosis. J. Pharmacol. Exp. Ther. 219: 299, 1981.

2. Bergquist, D., Bjorck, C. G., Dougan, P., Esquivel, C. O., Lannerstad, D., Nilsson, B., Saldeen, P., and Saldeen, T. The effect of inhibition of thromboxane synthesis in experimental thrombosis and hemostasis. Thromb. Res. 37: 435, 1985.

3. Bomberger, R. A., DePalma, R. G., Ambrose, T. A., and Manalo, P. Aspirin and dipyridamole inhibit endothelial healing. Arch. Surg. 117: 1459, 1982.

4. Buchanan, M. R., DeJana, E., Gent, M., Mustard, J. F., and Hirsch, J. Enhanced platelet accumulation onto injured carotid arteries in rabbits after aspirin treatment. J. Clin. Invest. 67: 503, 1981.

5. Bush, H. L., Hong, S. I., Deyken, D., and Nabseth, D. C. Effect of surgical trauma on prostacyclin production by vein grafts. Surg. Forum 33: 463, 1982.

6. Chesebro, J. H., Fusler, V., Elveback, L. R., Clements, I. P., Smith, H. C., Holmes, D. R., Jr., Bardsley, W. T., Pluth, J. R., Wallace, R. B., Puger, F. J., Orszulak, T. A., Piehler, J. M., Danielson, G. K., Schaff, H. V., and Frye, R. L. Effect of dipyridamole and aspirin on late vein-graft patency after coronary bypass operations. N. Engl. .I. Med. 310: 209, 1984.

7. Cronenwett, J. L., and Shellito, J. L. Effect of intraarterial carbacyclin infusion on platelet deposition in canine polytetrafluoroethylene grafts. Surg. Forum 36: 439, 1985.

8. Dale, J., Thaulow, E., Myhre, E., and Parry, J. The effect of a thromboxane synthetase inhibitor, dazoxiben, and acetylsalicylic acid on platelet function and prostaglandin metabolism. Thromb. Haemostasis 50: 703, 1983.

9. Daniel, W. W. Applied Nonparametric Statistics. Boston: Houghton Mifflin, 1978.

10. Davies, J. A., and Menys, V. C. Effect of dazoxiben on platelet-vessel wall interaction. Brit. J. Clin. Pharmacol. 15: 45S, 1983.

11. Defreyn, G., Deckmyn, H., and Vermylen, J. A thromboxane synthetase inhibitor reorients endoperoxide metabolism in whole blood towards prostacyclin and prostaglandin $\mathrm{E}_{2}$. Thromb. Res. 26: 389, 1982.
12. Eldor, A., Falcone, D. J., Hajjar, D. P., Minick, C. R., and Weksler, B. B. Recovery of prostacyclin production by de-endothelialized rabbit aorta. J. Clin. Invest. 67: 735, 1981.

13. Fitzpatrick, F. A., Borman, R. R., McGuire, J. C., Kelley, R. C., Wynalda, M. A., and Sun, F. F. A radioimmunoassay for thromboxane $\mathrm{B}_{2}$. Anal. Biochem. 82: 1,1977

14. Goldman, M., Hall, C., Hawker, R. J., and McCollum, C. N. Dazoxiben examined for platelet inhibitory effect in an artifical circulation. Brit. J. Clin. Pharmacol. 15: $615,1983$.

15. Gorman, R. R., Johnson, R. A., Spilman, C. H., and Aiken, J. W. Inhibition of platelet thromboxane $A_{2}$ synthetase activity by sodium 5-(3'-pyridinylmethyl) benzofuran-2-cayboxylate. Prostaglandins 26: 325 , 1983.

16. Hall, E. R., Chen, Y. C., Ho, T., and Wu, K. K. The reduction of platelet thrombi on damaged vessel wall by a thromboxane synthetase inhibitor in rabbits. Thromb. Res. 27: 501, 1982.

17. Hanson, S. R., and Harker, L. A. Effect of dazoxiben on arterial graft thrombosis in the baboon. Brit. $J$. Clin. Pharmacol. 15: 57S, 1983.

18. LoGerfo, F. W., Quist, W. C., Cantelmo, N. L., and Haudenschild, C. C. Integrity of vein grafts as a function of initial intimal and medial preservation. Circulation 68(Suppl. II): 117, 1983.

19. Kohler, T. R., Kaufman, J. L., Kacoyanis, G., Clowes, A., Donaldson, M. C., Kelly, E., Skillman, J., Couch, N. P., Whitemore, A. D., Mannick, J. A., and Salzman, E. W. Effect of aspirin and dipyridamole on the patency of lower extremity bypass grafts. Surgery 96: $462,1984$.

20. McCann, R. L., Hagen, P.-O., and Fuchs, J. C. A. Aspirin and dipyridamole decrease intimal hyperplasia in experimental vein grafts. Ann. Surg. 191: 238, 1980.

21. Randall, M. J., and Wilding, R. I. R. Acute arterial thrombosis in rabbits: Reduced platelet accumulation after treatment with dazoxiben hydrochloride (UK 37,248-01). Brit. J. Clin. Pharmacol. 15: 495, 1983.

22. Serneri, G. G. N., Abbate, R., Gensini, G. F., Panetta, A., Casolo, G. C., and Carini, M. TXA 2 production by human arteries and veins. Prostaglandins 25: 753 , 1983.

23. Thakur, M. L., Walsh, L., Malech, H. L., and Goltschalk, A. Indium-111 labclcd human platelets: Improved method, efficacy, and evaluation. J. Nucl. Med. 22: 381, 1981.

24. Wynalda, M. A., Liggett, W. F., and Fitzpatrick, F. A. Sodium 5-(3'-pyridinylmethyl) benzofuran-2carboxylate (U-63557A), a new, selective thromboxane synthase inhibitor: Intravenous and oral pharmacokinetics in dogs and correlations with ex situ thromboxane $\mathbf{B}_{2}$ production. Prostaglandins 26: 311 , 1983. 